\title{
Clinical course and urinary eicosanoids in patients with aspirin-induced urticaria followed up for 4 years
}

\author{
Malgorzata Setkowicz, MD, PhD, ${ }^{a}$ Lucyna Mastalerz, MD, PhD, ${ }^{b}$ Magdalena Podolec-Rubis, MD, PhD, ${ }^{a}$ \\ Marek Sanak, MD, PhD, ${ }^{\mathbf{b}}$ and Andrzej Szczeklik, MD, PhD ${ }^{\mathbf{b}} \quad$ Krakow, Poland
}

Background: Little is known about the course of aspirininduced urticaria. A special regulatory role of cysteinyl leukotrienes and prostaglandin $\mathrm{D}_{2}\left(\mathrm{PGD}_{2}\right)$ has been postulated. Objective: We performed a long-term observation on clinical course, aspirin sensitivity, and urinary eicosanoids in patients with aspirin-induced urticaria.

Methods: For 4 years, we followed up 22 patients with chronic idiopathic urticaria and aspirin hypersensitivity who restrained from the use of aspirin and other COX-1 inhibitors. Aspirin challenges were performed in 2002 (all results were positive) and repeated in 2006. Levels of urinary leukotriene $\mathbf{E}_{4}\left(\mathrm{LTE}_{4}\right)$ and the main $\mathrm{PGD}_{2}$ metabolite, $9 \alpha 11 \beta \mathrm{PGF}_{2}$, were measured at the same time points.

Results: During the follow-up period, the severity of urticaria has decreased. In 14 of 22 patients, the results of aspirin challenge remained positive. In 2002, these 14 patients responded to aspirin with a significant increase in urinary $\mathrm{LTE}_{4}$ and $9 \alpha 11 \beta P_{G} F_{2}$ levels. When studied 4 years later, they showed a similar response of $9 \alpha 11 \beta \mathrm{PGF}_{2}(P=.047)$ and a tendency toward an increase in $\mathrm{LTE}_{4}$ level $(P=.057)$. There was a correlation between the urinary $\mathrm{LTE}_{4}$ concentration after aspirin challenge and the intensity of skin eruptions. The dose of aspirin had no effect on the magnitude of response of both $\mathrm{LTE}_{4}$ and the $\mathrm{PGD}_{2}$ metabolite. In the remaining 8 patients, negative aspirin challenge results were not associated with changes in the urinary eicosanoids studied.

Conclusions: Aspirin hypersensitivity manifesting as urticaria/ angioedema remains present after 4 years in about two thirds of patients. Aspirin-precipitated skin reactions associate with increased excretion of $\mathrm{LTE}_{\mathbf{4}}$ and $\mathrm{PGD}_{2}$. (J Allergy Clin Immunol 2009;123:174-8.)

Key words: Urticaria, aspirin-induced urticaria, aspirin sensitivity, eicosanoids, leukotriene $\mathrm{E}_{4}$, prostaglandin $\mathrm{D}_{2}$

Some patients with chronic idiopathic urticaria have wheals and even angioedema after aspirin administration. In others aspirin causes an obvious increase in the underlying urticaria.

\footnotetext{
From the Departments of ${ }^{\mathrm{a}}$ Dermatology and ${ }^{\mathrm{b}}$ Medicine, Jagiellonian University School of Medicine.

Disclosure of potential conflict of interest: The authors have declared that they have no conflict of interest.

Received for publication July 18, 2008; revised September 2, 2008; accepted for publication September 3, 2008.

Available online October 9, 2008.

Reprint requests: Andrzej Szczeklik, MD, PhD, Department of Medicine, Jagiellonian University School of Medicine, ul. Skawińska 8, 31-066 Krakow, Poland. E-mail: mmszczek@cyf-kr.edu.pl.

0091-6749/\$36.00

(C) 2009 American Academy of Allergy, Asthma \& Immunology

doi:10.1016/j.jaci.2008.09.005
}

\author{
Abbreviations used \\ AIU: Aspirin-induced urticaria \\ LTE$_{4}$ : Leukotriene $\mathrm{E}_{4}$ \\ PASI: Psoriasis Area and Severity Index \\ $\mathrm{PGD}_{2}$ : Prostaglandin $\mathrm{D}_{2}$ \\ $9 \alpha 11 \beta \mathrm{PGF}_{2}$ : Prostaglandin $\mathrm{D}_{2}$ metabolite
}

The reaction can occur in just 15 minutes or up to 24 hours after aspirin ingestion, but on average, it develops within 1 to 4 hours. Most cases resolve within a few hours, but in severe reactions bouts of multiform skin eruptions can continue for 10 days after aspirin intake. $^{1-7}$

The histologic spectrum of cutaneous reactions to aspirin ${ }^{8}$ is dominated by a classic pattern of urticarial tissue. Occasionally, an interstitial fibrohistiocytic reaction pattern can develop. In sporadic patients, a perivascular dermal lymphocyte infiltrate or paucicellular mucinosis was observed.

Recent evidence indicates that the mechanism of aspirininduced urticaria (AIU) is related to inhibition of COX-1. ${ }^{9}$ This explains the cross-reactivity of various COX-1 inhibitors with different chemical structure. In contrast, COX-2 inhibitors are generally well tolerated by patients with AIU. ${ }^{10-13}$ Eicosanoid alterations, similar to those observed in patients with aspirin-induced asthma, were reported in patients with AIU. ${ }^{9}$ Specifically, in both clinical syndromes, aspirin challenges were accompanied by an increase in cysteinyl leukotriene and prostaglandin $\mathrm{D}_{2}$ $\left(\mathrm{PGD}_{2}\right)$ levels. AIU aggregates in families inheriting the leukotriene $\mathrm{C}_{4}$ variant. ${ }^{14}$ This segregation does not follow a clear Mendelian pattern. The human leukocyte antigen DRD1*1302DQB1*0609-DPB1*0201 haplotype was found to be a strong genetic marker in the Korean population. ${ }^{15}$

AIU can be a long-lasting ailment. However, little is known about the duration and perseverance of the sensitivity, as well as the eicosanoid alterations that accompany it. This prompted the study here reported, in which we followed up a well-characterized group of patients over a period of 4 years.

\section{METHODS \\ Patients}

The study group consisted of 22 patients with chronic idiopathic urticaria and aspirin sensitivity. They were recruited from a population of 30 patients with urticaria who in the year 2002 had positive aspirin challenge results and measurement of eicosanoid urinary metabolites. The remaining 8 patients from the original population were either lost or did not agree to participate.

The 22 patients were asked to use diet without artificial flavoring and preservatives and avoid aspirin and other COX-1 inhibitors. They were followed 
TABLE I. Patients' characteristic and parameters studied in all subjects $(n=22)$ and in subgroups with positive ( $\mathrm{n}=14$ ) and negative $(\mathrm{n}=8)$ aspirin challenge results separately

AIU (n = 22)

\section{Age (y)}

Female/male sex

Duration of urticaria (y)

Urinary $\mathrm{LTE}_{4}$ levels at

baseline ( $\mathrm{pg} / \mathrm{mg}$ creatinine)

Urinary $9 \alpha 11 \beta \mathrm{PGF}_{2}$ at baseline ( $\mathrm{ng} / \mathrm{mg}$ creatinine)

Total IgE (IU/mL)

Blood eosinophil count
$49.0 \pm 12.5,53(40-56)$

$16 / 6$

$12.2 \pm 9.5,7.0(5-19)$

$463.8 \pm 332.3,419.0(313.0-495.5)$

$0.380 \pm 0.145,0.367(0.275-0.500)$

$184.3 \pm 257.4,116.5(39.1-218)$

$225.8 \pm 224.5,181(90-293)$
$\mathrm{AlU}++(\mathrm{n}=14)$

$47.6 \pm 11.3,52.5(40.0-55.0)$

$11 / 3$

$11.4 \pm 7.1,7.5(5.0-19.0)$

$485.0 \pm 411.8,406.8$ (295.5-550.0)

$0.395 \pm 0.146 .0,0.363(0.280-0.515)$

$118 \pm 77.0,116(39.1-193.0)$

$239.7 \pm 247.1,212(90.0-303.0)$
AlU+- (n = 8)

$51.5 \pm 14.8,54.5$ (44.5-61.0)

$5 / 3$

$13.5 \pm 13.0,6.0(4.8-24.0)$

$426.8 \pm 117.3,422.5(326.3-479.8)$

$0.353 \pm 0.150,0.367(0.210-0.485)$

$299.4 \pm 404.8,157.0(32.5-390.5)$

$201.4 \pm 191.8,167$ (72.0-257.5)

AIU, Patients with aspirin-induced urticaria with a positive aspirin test result in the year 2002; AIU++, patients with a positive aspirin test result twice in the 2002 and 2006; $A I U+-$, patients with a positive aspirin test result in 2002 and a negative result in 2006.

Values are expressed as the mean $\pm \mathrm{SD}$ and median (25th and 75 th percentiles). Baseline values of eicosanoids in patients in the AIU, AIU ++ , and AIU +- groups are shown (values represent means of 2 estimations performed on placebo and aspirin days). There was no statistical difference between the subgroups in the parameters presented in the table.
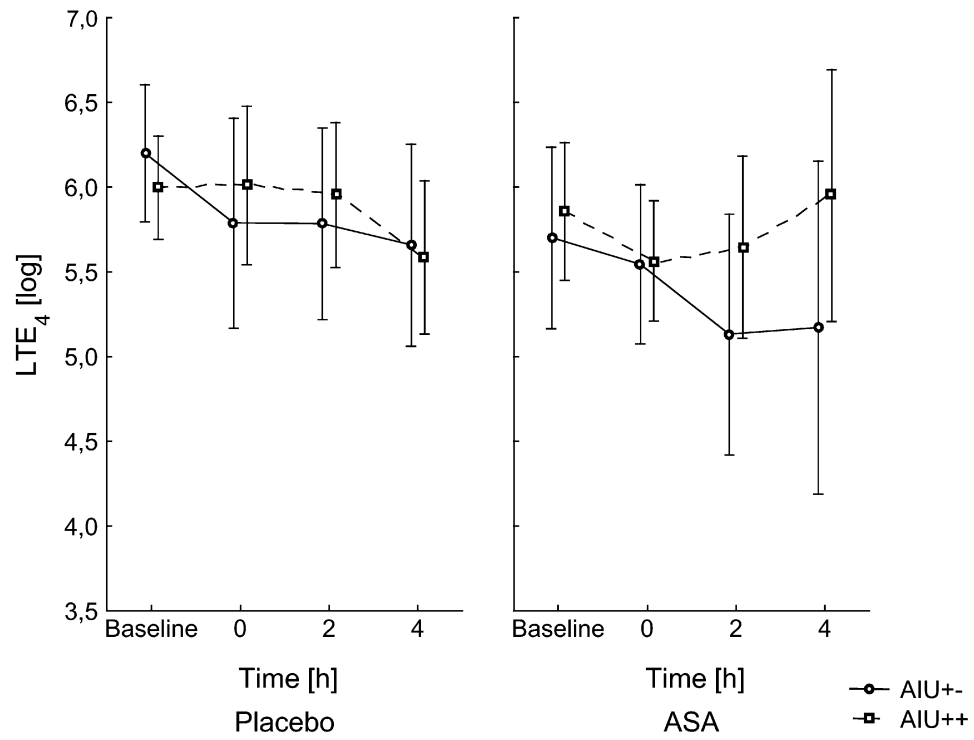

FIG 1. $\mathrm{LTE}_{4}$ urinary values (mean $\pm \mathrm{SD}$ ) after challenge with placebo or aspirin in the 2 groups studied $(\mathrm{AIU}++$ and $\mathrm{AIU}+-)$. For explanation, see the text.

up at the outpatient clinic at least twice a year by the same physician, and history, symptoms, and treatment were recorded. Four years later, all patients underwent aspirin challenge and had levels of urinary eicosanoids measured.

An aspirin oral challenge test was performed twice, in 2002 and 2006, by using the same protocol ${ }^{9}$ in the same laboratory. The test was carried out on 2 consecutive days: on day 1 , placebo was administered, and on day 2 , aspirin was administered. Before the test, the medications were withheld according to international guidelines. ${ }^{16}$ In patients who had positive aspirin challenge results in 2002 and 2006 (AIU++), urine samples were collected for measurement of leukotriene $\mathrm{E}_{4}\left(\mathrm{LTE}_{4}\right)$ and $9 \alpha 11 \beta \mathrm{PGF}_{2}\left(\mathrm{a} \mathrm{PGD}_{2}\right.$ metabolite) levels at baseline, at the time of appearance of the skin symptoms (time 0 ), and then 2 and 4 hours later. In patients with a positive result on aspirin challenge in 2002 and a negative result in 2006 (AIU+-), urine samples, in case of absent clinical reaction, were collected at baseline, 1 hour after the last aspirin dose (ie, when the cumulative dose of $500 \mathrm{mg}$ of aspirin was reached [time 0]), and then 2 and 4 hours later.

\section{Urinary eicosanoid determinations}

Urinary $\mathrm{LTE}_{4}$ was measured in unpurified urine samples by means of direct enzyme immunoassay (Cayman Chemical, Ann Arbor, Mich), following the same procedure as in the year $2002 .{ }^{9}$ Levels of $\mathrm{LTE}_{4}$ were expressed in picograms per milligram of creatinine.

The urinary concentration of $9 \alpha 11 \beta \mathrm{PGF}_{2}$ was measured by means of gas chromatography-negative ion chemical ionization-mass spectrometry (Hewlett-Packard, Palo Alto, Calif) by using the same procedure used in 2002. ${ }^{9}$ Results were expressed in nanograms per milligram of creatinine.

\section{Assessment of the severity of skin eruption}

A modified Psoriasis Area and Severity Index (PASI) score was used to standardize the assessment of the severity of skin eruptions during aspirin challenge tests. For the assessment of urticaria, itching replaced desquamation in the index. ${ }^{9}$ The determinations were carried out by an experienced dermatologist at the time of the first appearance of skin lesions and 2, 4, and 6 hours later.

\section{Lung function}

Pulmonary function tests were performed on a flow-integrating computerized pneumotachograph (Pneumoscreen; E. Jaeger, Würzburg, Germany). 

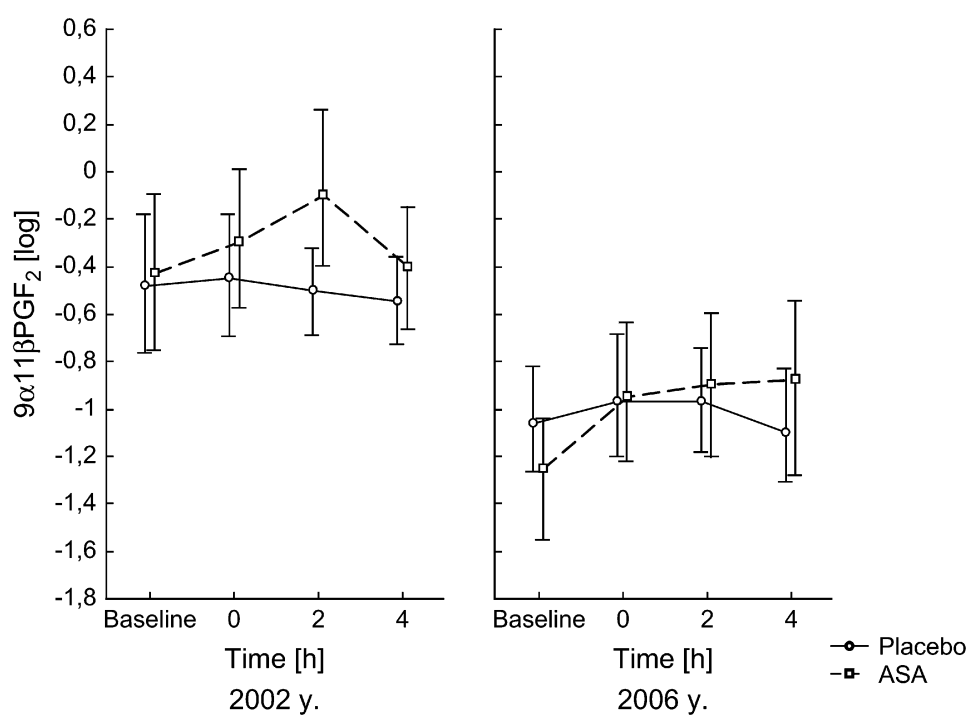

FIG 2. Response of urinary $9 \alpha 11 \beta \mathrm{PGF}_{2}$ to placebo or aspirin (ASA) challenge in the whole group of 22 patients in 2002 and 2006.

\section{Statistical analysis}

Summary statistics were expressed as the mean and SD for symmetrically distributed data or the geometric mean and $25 \%$ and $75 \%$ percentiles for nonsymmetrically (skewed) distributed data. The multiway ANOVA model was used for multiple-group comparisons. Logarithmic transformation was used when needed as a variance-stabilizing transformation. The Fisher exact test was used for dichotomous data for 2 independent random samples. A $P$ value of .05 or less was considered statistically significant.

\section{RESULTS}

Over the period of 4 years, hives, occasionally accompanied by swelling of the face and hands unrelated to nonsteroidal antiinflammatory drugs, occurred in all patients. The number of patients experiencing episodes of urticaria decreased; in the first year, 22 patients had bouts of urticaria, and in the fourth year, only 12 had urticaria. The intensity of spontaneous urticaria, as well as its frequency, decreased. The number of patients who required long-term medical treatment also decreased. Thus the secondgeneration antihistamines were used during the first year in 9 patients, but during the fourth year, they were used only in 2 patients; a tricyclic antidepressant (doxepin) was initially used in 2 patients but in none in the fourth year. Five patients were treated with systemic steroids during the first year, but none were so treated at the end of the study.

\section{Oral aspirin provocation test}

None of the patients had symptoms after administration of placebo. In $14(63.6 \%)$ of 22 patients, the results of aspirin challenge tests were positive. Those patients had skin rash, angioedema, or both, but dyspnea was absent, and spirometric values remained stable throughout the observation period. There were no statistical differences in clinical characteristics between the patients who had positive aspirin challenge results $(n=14$, $\mathrm{AIU}++)$ and those who tolerated aspirin well $(\mathrm{n}=8$, AIU+-; Table I).

In 2006, none of the patients had skin reactions after a 71-mg cumulative dose of aspirin. After $188 \mathrm{mg}$, urticaria/angioedema developed in 6 subjects, and after $500 \mathrm{mg}$, it developed in 8 subjects. Patients with a lower cumulative dose of aspirin $(\leq 188 \mathrm{mg})$ in $2002 \mathrm{had}$ more frequently positive aspirin challenge results in the year $2006(P=.046)$. The severity of their skin eruptions after aspirin challenge (PASImax score) was lower than 4 years before $(P=.02$, ANOVA).

\section{Urinary eicosanoids}

Baseline levels. Comparison of baseline urinary $\mathrm{LTE}_{4}$ levels in 2006 and 2002 showed no difference in either the whole group $(\mathrm{n}=22)$ or in the subgroups studied $(P>.05$, data not shown). However, the baseline $9 \alpha 11 \beta \mathrm{PGF}_{2}$ levels in 2002 were significantly higher than in 2006 in the total group of 22 patients $(0.796 \pm 0.471$ vs $0.366 \pm 0.171 \mathrm{ng} / \mathrm{mg}$ creatinine, $P<0.001)$, as well as in the subgroup of AIU ++ patients $(0.876 \pm 0.485$ vs $0.347 \pm 0.164 \mathrm{ng} / \mathrm{mg}$ creatinine; $P<.003$ ).

Aspirin challenge. Baseline urinary $\mathrm{LTE}_{4}$ excretion (Table I) did not differ between patients with positive $(\mathrm{n}=14$, AIU ++$)$ and negative $(\mathrm{n}=8$, AIU +-$)$ aspirin challenge results $(P=$ .92). After placebo, urinary $\mathrm{LTE}_{4}$ levels gradually decreased in both study groups $(P=.002$, ANOVA), a phenomenon observed also 4 years earlier. Urinary LTE $_{4}$ levels have a tendency to increase after aspirin challenge tests compared with baseline values $(P=.057)$ only in the group of patients $(\mathrm{AIU}++)$ with positive aspirin challenge results (Fig 1). After challenge, urinary $\mathrm{LTE}_{4}$ levels were the highest in patients with severe skin reactions. There was a correlation between the urinary levels of LTE $_{4} 4$ hours after aspirin challenge and the maximal intensity of skin eruption expressed as the PASI score (Spearman $r=0.64, P=$ $.01)$. The dose of aspirin had no effect on the magnitude of the response of $\mathrm{LTE}_{4}$.

In 2006 , the response of $9 \alpha 11 \beta \mathrm{PGF}_{2}$ to aspirin challenge was similar to that recorded in 2002, although the baseline values were distinctly lower and the response less accentuated (Fig 2). Analysis of the subgroups based on the presence or absence of clinical symptoms revealed that placebo administration produced no significant differences in the urinary $\mathrm{PGD}_{2}$ metabolite levels in either group $(P=.25$, ANOVA). After aspirin challenge, urinary concentrations of $9 \alpha 11 \beta \mathrm{PGF}_{2}$ increased significantly only in the 
group of patients who had a clinical reaction. They reached their peak at the moment of clinical reaction (increase from $0.347 \pm$ 0.164 to $0.516 \pm 0.352 \mathrm{ng} / \mathrm{mg}$ creatinine, $P=.047$ ) and returned to baseline values 4 hours later. The dose of aspirin had no effect on the magnitude of the response of $9 \alpha 11 \beta \mathrm{PGF}_{2}$. In the AIU+subgroup $\mathrm{PGD}_{2}$ metabolites after aspirin showed a tendency to increase, which did not reach statistical significance $(P=.086$, ANOVA).

\section{DISCUSSION}

We had an opportunity to follow-up a group of patients with well-documented AIU over a period of 4 years. After 4 years, we could also retest their sensitivity to aspirin using the oral provocation test, as well as again measure urinary eicosanoid metabolites, which showed distinct alterations at entry to the study. The advantage of the study was that the patients were followed up by the same physician, and both provocation tests and biochemical measurements were performed at the same laboratory by using the same methods.

Over the period of 4 years, the intensity of urticaria decreased. This was reflected by a decrease in the severity and frequency of spontaneous urticarial eruptions, a decrease in hospitalizations, and a progressively lower number of antihistamines and corticosteroids prescribed. The reason for this improvement could have been total avoidance of aspirin and other drugs that inhibit COX1 and perhaps also the elimination diet. Regular clinical care was probably another important factor.

Despite a distinct clinical improvement, about two thirds of the patients remained hypersensitive to aspirin, and the results of the provocation tests were definitely positive in them. It is of interest that one of these patients, who reacted 4 years earlier to $188 \mathrm{mg}$ of aspirin not only with skin eruptions but also with rhinorrhea, conjunctivitis, shortness of breath, and a decrease in $\mathrm{FEV}_{1}$ of less than $20 \%$, now responded only with a skin reaction after challenge with $500 \mathrm{mg}$. Elimination of aspirin sensitivity has been reported in aspirin-induced asthma, ${ }^{16}$ but it is a rare phenomenon. We cannot exclude the possibility that the higher dose of aspirin, exceeding $500 \mathrm{mg}$, would have precipitated bronchial or skin reactions in our patients.

In subjects who remained hypersensitive to aspirin, the aspirin provocation test produced eicosanoid responses similar to those 4 years earlier. The magnitude of the response was, however, less accentuated, which could be linked to the gradual improvement in the patients' clinical state. Thus in all patients aspirin challenge resulted in clinical reaction and an increase in urinary $\mathrm{LTE}_{4}$ levels in contrast to the group of 8 patients who lost their hypersensitivity to aspirin. The urinary $\mathrm{LTE}_{4}$ value reached the highest levels in subjects with the most severe skin reactions. The dose of aspirin used, however, had no effect on the clinical response, a phenomenon already observed earlier. ${ }^{9}$ Finally, urinary levels of a stable $\mathrm{PGD}_{2}$ metabolite showed significant changes only in the group of patients with positive aspirin challenge results.

Several explanations can be offered for the blunted reactivity to aspirin in our patients after the 4-year follow-up period. Chronic idiopathic urticaria itself has a variable clinical course. Elimination of aspirin and other COX-1 inhibitors might have lessened the predisposition to the reactions. An unknown causative factor responsible for aspirin hyperreactivity could also stop the reactions. Eventually, autoimmune abnormalities present in a substantial fraction of patients with idiopathic urticaria, $1,4,17,18$ including those with aspirin sensitivity, ${ }^{19,20}$ could subside. From our long-term observation, the mechanism involved in urticarial eruption seems to correlate well with the clinical status of the patient. Systemic overproduction of $\mathrm{PGD}_{2}$ metabolites and cysteinyl leukotrienes, triggered by aspirin, predicts a phenotype of AIU and seems to be a biologic hallmark of the inflammatory mechanism involved. Thus aspirin and other drugs sharing a similar pharmacologic profile are required to reveal underlying biochemical abnormalities of arachidonic acid cascade. Perhaps aspirin, by depleting protective $\mathrm{PGE}_{2}$, promotes unrestrained synthesis of cysteinyl leukotrienes and release of such mediators as $\mathrm{PGD}_{2}$ from mast cells. In aspirin-induced asthma this seems to be a likely mechanism. ${ }^{21} \mathrm{PGD}_{2}$ is the major COX metabolite almost entirely generated by activated mast cells. ${ }^{22}$ In bronchial asthma mast cells infiltrate airway smooth muscle, a phenomenon associated with high $\mathrm{PGD}_{2}$ levels in the sputum. ${ }^{23}$ Decreased reactivity of the skin mast cells or a decrease in their number is the plausible, although hypothetical, explanation for both clinical improvement of urticaria and depression of aspirin sensitivity in our patients. Thus, reduction in skin mast cell reactivity is accompanied by a return of biosynthesis of proinflammatory mediators of arachidonic acid to normal levels.

\section{Key messages}

- Aspirin hypersensitivity manifesting as urticaria/angioedema remains present after 4 years in about two thirds of patients.

- The aspirin-triggered skin reactions are accompanied by a tendency toward overproduction of cysteinyl leukotrienes and $\mathbf{P G D}_{2}$.

\section{REFERENCES}

1. Kaplan AP. Clinical practice. Chronic urticaria and angioedema. N Engl J Med 2002;346:175-9.

2. Kaplan AP. Chronic urticaria: pathogenesis and treatment. J Allergy Clin Immunol 2004;114:465-74

3. Grattan CE, Humphreys F. British Association of Dermatologists Therapy Guidelines and Audit Subcommittee. Guidelines for evaluation and management of urticaria in adults and children. Br J Dermatol 2007;157:1116-23.

4. Greaves MW, Tan KT. Chronic urticaria: recent advances. Clin Rev Allergy Immunol 2007;33:134-43.

5. Zuberbier T, Bindslev-Jensen C, Canonica W, Grattan CE, Greaves MW, Henz $\mathrm{BM}$, et al. EAACI/GA2LEN/EDF guideline: management of urticaria. Allergy 2006;61:321-31

6. Stevenson DD, Simon RA, Zuraw BL. Sensitivity to aspirin and nonsteroidal antiinfalmmatory drugs. In: Middelton's allergy, principles and practice. 6th ed. St Louis: Mosby, Inc; 2003. p. 1695-710.

7. Grzelewska-Rzymowska I, Rozniecki J, Szmidt M. Aspirin desensitization in patients with aspirin-induced urticaria and angioedema. Allergol Immunopathol 1988; $16: 305-8$

8. Zembowicz A, Mastalerz L, Setkowicz M, Radziszewski W, Szczeklik A. Histological spectrum of cutaneous reactions to aspirin in chronic idiopathic urticaria. J Cutan Pathol 2004;31:323-9.

9. Mastalerz L, Setkowicz M, Sanak M, Szczeklik A. Hypersensitivity to aspirin: common eicosanoid alterations in urticaria and asthma. J Allergy Clin Immunol 2004;113:771-5

10. Zembowicz A, Mastalerz L, Setkowicz M, Radziszewski W, Szczeklik A. Safety of cyclooxygenase II inhibitors and increased leukotriene synthesis in chronic idiopathic urticaria with sensitivity to non-steroidal anti-inflammatory drugs. Arch Dermatol 2003; 139:1577-82.

11. Ye YM, Kim JE, Kim SH, Suh CH, Nam DH, Park HS. Clinical characteristics of chronic urticaria with aspirin sensitivity. Korean J Asthma Allergy Clin Immunol $2005 ; 25: 194-9$ 
12. Sanchez-Borges M, Capriles-Hulett A, Caballero-Fonseca F. How safe are COX-2 inhibitors in NSAIDs-sensitive subjects with cutaneous reactions? ACI Int 2003; 15:110-3.

13. Asero R. Etoricoxib challenge in patients with chronic urticaria with NSAID intolerance. Clin Exp Dermatol 2007;32:661-3.

14. Mastalerz L, Setkowicz M, Sanak M, Rybarczyk H, Szczeklik A. Familial aggregation of aspirin-induced urticaria and leukotriene $\mathrm{C}$ synthase allelic variant. $\mathrm{Br} \mathrm{J}$ Dermatol 2006;154:256-60.

15. Kim SH, Choi JH, Lee KW, Kim SH, Shin ES, Oh HB, et al. The human leucocyte antigen-DRB1*1302-DQB1*0609-DPB $1 * 0201$ haplotype may be a strong genetic marker for aspirin-induced urticaria. Clin Exp Allergy 2005;35: 339-44.

16. Nizankowska-Mogilnicka E, Bochenek G, Mastalerz L, Swierczyńska M, Picado C, Scadding G, et al. EAACI/GA2LEN guideline: aspirin provocation tests for diagnosis of aspirin hypersensitivity. Allergy 2007;62:1111-8.

17. Hide M, Francis DM, Grattan CE, Hakimi J, Kochan JP, Greaves MW. Autoantibodies against the high-affinity IgE receptor as a cause of histamine release in chronic urticaria. N Engl J Med 1993;328:1599-604.
18. Fiebiger E, Maurer D, Holub H, Reininger B, Hartmann G, Woisetschläger M, et al. Serum IgG autoantibodies directed against the alpha chain of Fc epsilon RI: a selective marker and pathogenetic factor for a distinct subset of chronic urticaria patients? J Clin Invest 1995;96:2606-12.

19. Asero R, Tedeschi A, Lorini M. Autoreactivity is highly prevalent in patients with multiple intolerances to NSAIDs. Ann Allergy Asthma Immunol 2002;88:468-72.

20. Szczeklik A, Nizankowska E, Serafin A, Dyczek A, Duplaga M, Musial J. Autoimmune phenomena in bronchial asthma with special reference to aspirin intolerance. Am J Respir Crit Care Med 1995;152:1753-6.

21. Mastalerz L, Sanak M, Gawlewicz-Mroczka A, Gielicz A, Cmiel A, Szczeklik A. Prostaglandin E2 systemic production in patients with asthma with and without aspirin hypersensitivity. Thorax 2008;63:27-34.

22. Bochenek G, Nizankowska E, Gielicz A, Swierczyńska M, Szczeklik A. Plasma 9alpha,11beta-PGF2, a PGD2 metabolite, as a sensitive marker of mast cell activation by allergen in bronchial asthma. Thorax 2004;59:459-64.

23. Brightling CE, Bradding P, Symon FA, Holgate ST, Wardlaw AJ, Pavord ID. Mastcell infiltration of airway smooth muscle in asthma. N Engl J Med 2002;346: 1699-705.

\section{Receive tables of contents by e-mail}

To receive tables of contents by e-mail, sign up through our Web site at www.jacionline.org

\section{Instructions}

Log on and click "Register" in the upper right-hand corner. After completing the registration process, click on "My Alerts" then "Add Table of Contents Alert." Select the specialty category "Allergy"

or type The Journal of Allergy and Clinical Immunology in the search field and click on the Journal title. The title will then appear in your "Table of Contents Alerts" list.

Alternatively, if you are logged in and have already completed the Registration process, you may add tables of contents alerts by accessing an issue of the Journal and clicking on the "Add TOC Alert" link.

You will receive an e-mail message confirming that you have been added to the mailing list. Note that tables of contents e-mails will be sent when a new issue is posted to the Web. 